\title{
A pharmacoeconomic study of different brands of commonly prescribed antihypertensive drugs
}

\author{
Pooja C. Upasani, Rohidas M. Barve*, Rajesh S. Hiray
}

Department of Pharmacology, Byramjee Jeejeebhoy Government Medical College and Sassoon General Hospitals, Pune, Maharashtra, India

Received: 30 May 2021

Accepted: 24 June 2021

*Correspondence:

Dr. Rohidas M. Barve,

Email: rohidas.barve@yahoo.com

Copyright: $(\subset$ the author(s), publisher and licensee Medip Academy. This is an open-access article distributed under the terms of the Creative Commons Attribution Non-Commercial License, which permits unrestricted non-commercial use, distribution, and reproduction in any medium, provided the original work is properly cited.

\section{ABSTRACT}

Background: The hypertension is the most common chronic disease, therefore treatment should be affordable. The antihypertensive drugs of same strength are available in market at different costs. This study was undertaken to create awareness among health care workers and patients, about cost difference among different brands of same antihypertensive drug. So that whenever possible, a cheaper effective brand can be prescribed to ensure better patient adherence.

Methods: Maximum retail price (MRP) of various antihypertensive drugs of same strength, manufactured by different pharmaceutical companies was obtained from various offline and online sources. The minimum and maximum cost of 10 tablets/capsules noted. The cost ratio and percentage cost variation was calculated for single drug and fixed dose combinations. The ceiling price (as per DPCO) of essential antihypertensives (as per national list of essential medicines) was compared with their maximum cost.

Results: The formulations of single antihypertensive drugs (41) and fixed dose combinations of two drugs (19) and three drugs (9) were included in the study. Among the single antihypertensives analyzed the highest cost difference was of eplerenone $(50 \mathrm{mg})$ and high cost ratio and cost variation percentage was of amlodipine $(5 \mathrm{mg})$. Among fixed dose combination of two drugs analyzed highest cost difference was found that of hydrochlorothazide $(12.5 \mathrm{mg})+\mathrm{olmesartan}$ $(40 \mathrm{mg})$ combination and highest cost ratio and percentage cost variation was of amlodipine (5 mg)+telmisartan (40 $\mathrm{mg})$.

Conclusions: There was a huge price variation among the antihypertensive drugs manufactured by various companies. Some measures must be taken by the government to bring the uniformity in the price that will help to reduce the economic burden on the patients.

Keywords: Antihypertensive, Pharmacoeconomic, Cost variation, Upasani

\section{INTRODUCTION}

The burden of hypertension is progressively on a rise worldwide, with India contributing to a major part of this burden. The global burden of disease study stated that increase in systolic blood pressure is associated with the major burden among all risk factors, accounting for 10.2 million deaths and 208 million disability adjusted life years (DALYs). ${ }^{1}$
World health report 2002 identified hypertension as a 3rd ranked factor for DALYs. ${ }^{2}$ Hypertension is an important risk factor for chronic disease burden in India and a preventable contributor to mortality and morbidity and requires lifelong treatment. ${ }^{3,4}$ Nearly $10.8 \%$ of all deaths in India are attributed to hypertension. ${ }^{5}$ Several studies have indicated the decrease in the patient compliance is influence by drug prices. ${ }^{6}$ Thus, the therapeutic cost should be appropriate, affordable to each and every individual. 
In India most of the drugs are available by manufacturing brands and these are prescribed by clinicians mostly in brand names. This may affect the patients financial condition adversely if costly brand is prescribed especially in chronic diseases. ${ }^{7}$ Many chronically ill patients frequently cut back on medications owing to cost. Out-ofpocket costs and inadequate prescription coverage may lead to adherence problems for many important medication. There is wide cost variation among different brands of the same antihypertensive drug. Clinician's awareness of cost of therapeutics is poor. The costly brand of same generic drug is proved to be in no way superior to its economically cheaper counterpart. ${ }^{8}$

The national pharmaceutical pricing authority (NPPA) was established on 29 August 1997 to regulate the prices of pharmaceutical drugs in India. The implementation of the NPPA, 2012 and the drugs prices control order (DPCO), 2013 was brought about by NPPA. It safeguards the interest of both the manufacturer and the consumers by ensuring the availability of essential medicines at affordable prices. It fixed the ceiling prices of 856 formulations of medicines mentioned in the NLEM, 2015. Once medicine is brought under DPCO, it cannot be sold at a price higher than that fixed by the government. Also none of the combinations of antihypertensive drugs are included in DPCO list 2018. Many hypertensive patients need combination drug therapy during the course of the disease. Hence, it is desired that the government should bring all lifesaving drugs and combinations under price control. ${ }^{9,10}$ The common man therefore has to shell out more money with medicine prices spinning out of his reach.

During last few decades, the demand for healthcare has increased rapidly resulting in high expenditure. To spend financial resources as efficiently as possible, cost containment has assumed significant importance. ${ }^{11}$ Limited studies are available in Indian scenario, which compare the cost of drugs of different brands. Hence, this study was carried out to compare the cost of different brands of drugs used for treatment. The present study was aimed at investigating the cost differences in various brands of same antihypertensive drug, so that whenever possible, a cheaper effective brand could be prescribed.

\section{METHODS}

MRP of a particular antihypertensive drug (cost per 10 tablets/capsules) with the same strength and dosage forms manufactured by different pharmaceuticals was obtained. The data was retrieved from online sources including: Pharma Sahi Dam of NPPA of government of India, (NPPA) and CIMS (current index of medical specialties). The cost was cross-checked at pharmacy shops (retail drug stores).

The fixed dose combinations (FDC) of antihypertensive drugs were also analyzed for the cost differences. The FDC is the formulation including two or more active pharmaceutical ingredients combined in a single dosage form. FDCs included in the study were formulations containing two or three active ingredients combined in single dosage form.

The parameters included in this study were: (1) the minimum and maximum cost: the minimum and the maximum cost in rupees (₹) of a particular antihypertensive drug manufactured by various pharmaceutical companies in the same strength were noted; (2) the cost ratio: it is the ratio of the cost of the costliest to cheapest brand of a drug which tells, how many times costliest brand costs more than the cheapest one; (3) percentage cost variation: it was calculated as follows, ${ }^{12}$

$\%$ cost variation $=\frac{\text { maximum } \cos t-\text { minimum cost }}{\text { minimum } \cos t} \times 100$.

The maximum cost of essential antihypertensives (as per NLEM) was compared with their ceiling price (as per DPCO). ${ }^{10,13}$

Ceiling prices of 10 tablets/capsules were calculated. The cost difference between maximum cost and ceiling price was analyzed.

\section{RESULTS}

All the commonly prescribed groups of antihypertensive drugs were included in the present study. Wide cost variation was found in most of the antihypertensive drugs studied. We have studied 41 individual antihypertensive drugs. FDCs analyzed included two drug combination and three drug combination. Number of two drug fixed dose combinations studied were 19 and those of three drug combinations were 9 . The cost difference, cost ratio and percentage cost variation of all antihypertensive drugs were calculated.

\section{Diuretics}

Highest cost difference was seen with eplerenone $(50 \mathrm{mg}$ ) which was 311 and cost ratio and percentage cost variation were found to be highest with furosemide $(40 \mathrm{mg}$ ) (Table $1)$.

\section{Angiotensin converting enzyme inhibitors (ACEI)}

The highest cost difference was observed in case of lisinopril (10 mg) about 180, while highest cost ratio (9.32) and highest percentage cost variation (832.58) was with enalapril (10 mg) (Table 1).

\section{Angiotensin receptor blockers}

Among ARB's wide cost difference was found in case of valsartan (160 mg) (Table 1). Very high cost ratio (8.69) and percentage cost variation (769.64) were observed with losartan. 


\section{Sympathetic inhibitors}

Total 10 sympathetic inhibitors were analyzed in this study. Among them highest cost difference was observed with propranolol (10 $\mathrm{mg}$ ) and cost ratio and percentage cost variation were greater in case of metoprolol $(80 \mathrm{mg})$ as shown in Table 1.

\section{Calcium channel blockers}

Total 9 antihypertensive drugs from calcium channel blockers were analyzed. Maximum cost difference (120) was observed with benidipine $(8 \mathrm{mg})$. Cost ratio and percentage cost variation were higher in case of amolodipine ( $5 \mathrm{mg}$ ) brands (Table 1).

\section{Fixed dose combination (FDC)}

We compared 19 two drug FDCs and 9 three drug FDCs (Table 2 and 3 ).
Among two drug FDCs highest cost difference was found in case of different brands of hydrochlorothazide (12.5 $\mathrm{mg})+$ olmesartan $(40 \mathrm{mg})$ combination. Cost ratio and percentage cost variation were higher in case of amlodipine (5 mg)+telmisartan (40 mg) FDC brands (Table 2).

Three drugs combination of telmisartan $(40 \mathrm{mg})$, amlodipine $(5 \mathrm{mg})$ and hydrochlorothiazide $(12.5 \mathrm{mg}$ ) had highest cost difference and combination of olmisartan (20 $\mathrm{mg}$ ), amlodipine (5 mg) and hydrochlorothiazide (12.5 mg) has shown maximum cost ratio and percentage cost variation (Table 3).

The cost difference between maximum cost and the ceiling price of essential antihypertensive drugs was analyzed. The wide cost difference was found with amlodipine $(5 \mathrm{mg}$ ) means the maximum cost was far higher than the ceiling price. Most of the essential antihypertensives analyzed were having greater maximum cost as compared to the ceiling price. Only one essential antihypertensive drug (telmisartan) had maximum cost within the limit of the ceiling price (Table 4).

Table 1: Cost variation analysis of single drug formulations of antihypertensive drugs.

\begin{tabular}{|c|c|c|c|c|c|c|c|c|c|c|}
\hline $\begin{array}{l}\dot{\theta} \\
\dot{\dot{b}}\end{array}$ & है & 高 & 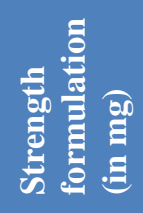 & 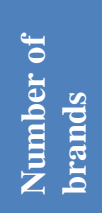 & 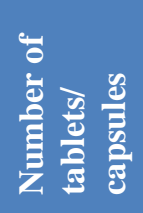 & $\begin{array}{l}\text { हू } \\
\text { ह } \\
\Xi\end{array}$ & 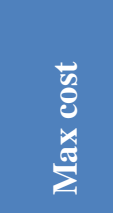 & 范 & 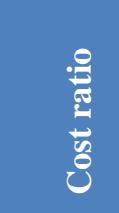 & 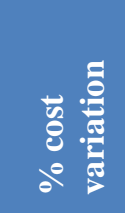 \\
\hline \multirow{4}{*}{1.} & \multicolumn{10}{|l|}{ Diuretics } \\
\hline & \multirow{3}{*}{ Hydrochlorothiazide } & \multirow{3}{*}{3} & 12.5 & 7 & 10 & 8.12 & 10.97 & 2.85 & 1.350 & 35.098 \\
\hline & & & 25 & 7 & 10 & 8.12 & 10.97 & 2.85 & 1.350 & 35.098 \\
\hline & & & 50 & 7 & 10 & 8.12 & 10.97 & 2.85 & 1.350 & 35.098 \\
\hline 2. & Chlorthalidone & 1 & 12.5 & 25 & 10 & 20 & 71 & 51 & 3.55 & 255 \\
\hline \multirow{2}{*}{3.} & \multirow{2}{*}{ Indapamide } & \multirow{2}{*}{2} & 1.5 & 2 & 10 & 69.5 & 100.6 & 31.1 & 1.447 & 44.748 \\
\hline & & & 2.5 & 4 & 10 & 44 & 107.1 & 63.1 & 2.4340 & 143.409 \\
\hline 4. & Furosemide & 1 & 40 & 16 & 10 & 3.3 & 36 & 32.7 & 10.909 & 990.909 \\
\hline \multirow{3}{*}{5.} & \multirow{3}{*}{ Spironolactone } & \multirow{3}{*}{3} & 25 & 5 & 10 & 19.35 & 31.8 & 12.45 & 1.643 & 64.341 \\
\hline & & & 50 & 4 & 10 & 25 & 50 & 25 & 2 & 100 \\
\hline & & & 100 & 3 & 10 & 42 & 191.9 & 149.9 & 4.569 & 356.904 \\
\hline \multirow{3}{*}{6.} & \multirow{2}{*}{ Eplerenone } & \multirow{2}{*}{2} & 25 & 13 & 10 & 140.3 & 354 & 213.7 & 2.523 & 152.316 \\
\hline & & & 50 & 9 & 10 & 287 & 598 & 311 & 2.083 & 108.362 \\
\hline & ACE inhibitors & & & & & & & & & \\
\hline 7. & Captopril & 1 & 25 & 3 & 10 & 25.98 & 41 & 15.02 & 1.578 & 57.813 \\
\hline \multirow{3}{*}{8.} & \multirow{3}{*}{ Enalapril } & \multirow{3}{*}{3} & 2.5 & 12 & 10 & 9.3 & 21.54 & 12.24 & 2.316 & 131.612 \\
\hline & & & 5 & 12 & 10 & 10 & 32.31 & 22.31 & 3.231 & 223.1 \\
\hline & & & 10 & 9 & 10 & 10.68 & 99.6 & 88.92 & 9.325 & 832.584 \\
\hline \multirow{3}{*}{9.} & \multirow{3}{*}{ Lisinopril } & \multirow{3}{*}{3} & 2.5 & 6 & 10 & 24.83 & 54 & 29.17 & 2.174 & 117.478 \\
\hline & & & 5 & 6 & 10 & 53.41 & 100 & 46.59 & 1.872 & 87.230 \\
\hline & & & 10 & 4 & 10 & 118 & 184 & 66 & 1.559 & 55.932 \\
\hline \multirow{2}{*}{10.} & \multirow{2}{*}{ Perindopril } & \multirow{2}{*}{2} & 4 & 3 & 10 & 88.75 & 135 & 46.25 & 1.521 & 52.112 \\
\hline & & & 8 & 2 & 10 & 119.75 & 170 & 50.25 & 1.419 & 41.962 \\
\hline 11. & Ramipril & 1 & 2.5 & 14 & 10 & 25.23 & 52.86 & 27.63 & 2.095 & 109.512 \\
\hline 12. & Fosinopril & 1 & 10 & 2 & 10 & 60.95 & 68.53 & 7.58 & 1.124 & 12.436 \\
\hline \multirow{2}{*}{13.} & Quinapril & 1 & 10 & 5 & 10 & 66.3 & 152.37 & 86.07 & 2.298 & 129.819 \\
\hline & ARB & & & & & & & & & \\
\hline
\end{tabular}




\begin{tabular}{|c|c|c|c|c|c|c|c|c|c|c|}
\hline $\begin{array}{l}\dot{z} \\
\dot{\bar{z}}\end{array}$ & 总 & 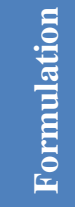 & 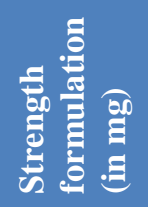 & 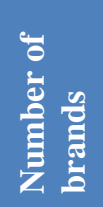 & 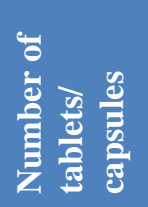 & 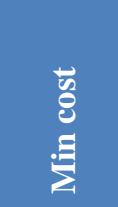 & 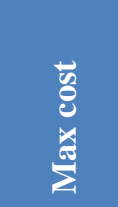 & 范 & 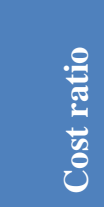 & 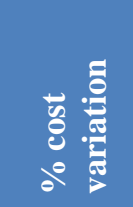 \\
\hline \multirow{2}{*}{14.} & \multirow{2}{*}{ Losartan } & \multirow{2}{*}{3} & 25 & 54 & 10 & 4.81 & 41.83 & 37.02 & 8.696 & 769.646 \\
\hline & & & 50 & 66 & 10 & 11.98 & 99 & 87.02 & 8.263 & 726.377 \\
\hline 15. & Candesartan & 1 & 16 & 7 & 10 & 61.65 & 120 & 58.35 & 1.946 & 94.647 \\
\hline \multirow{3}{*}{16.} & \multirow{3}{*}{ Valsartan } & \multirow{3}{*}{3} & 40 & 3 & 10 & 47.52 & 94.65 & 47.13 & 1.991 & 99.179 \\
\hline & & & $80 \mathrm{mg}$ & 5 & 10 & 86.3 & 165.35 & 79.05 & 1.915 & 91.599 \\
\hline & & & 160 & 3 & 10 & 129.2 & 345 & 215.8 & 2.670 & 167.027 \\
\hline \multirow{3}{*}{17.} & \multirow{3}{*}{ Telmisartan } & \multirow{3}{*}{3} & 20 & 113 & 10 & 15.92 & 61.5 & 45.58 & 3.863 & 286.306 \\
\hline & & & 40 & 113 & 10 & 13.75 & 59.6 & 45.85 & 4.334 & 333.454 \\
\hline & & & 80 & 113 & 10 & 15.92 & 61.5 & 45.58 & 3.863 & 286.306 \\
\hline \multirow{2}{*}{18.} & \multirow{2}{*}{ Irbesartan } & \multirow{2}{*}{2} & 150 & 2 & 10 & 155.5 & 213.5 & 58 & 1.372 & 37.299 \\
\hline & & & 300 & 2 & 10 & 243 & 286.8 & 43.8 & 1.180 & 18.024 \\
\hline \multirow{3}{*}{19.} & \multirow{3}{*}{ Olmesartan } & \multirow{3}{*}{3} & 10 & 10 & 10 & 55 & 102.8 & 47.8 & 1.869 & 86.909 \\
\hline & & & 20 & 69 & 10 & 29 & 128.3 & 99.3 & 4.424 & 342.413 \\
\hline & & & 40 & 61 & 10 & 49 & 226.5 & 177.5 & 4.622 & 362.244 \\
\hline \multicolumn{11}{|c|}{ Direct renin inhibitors } \\
\hline 20. & Aliskiren & 2 & 150 & 2 & 10 & 342.75 & 489.64 & $\begin{array}{l}146.8 \\
9\end{array}$ & 1.428 & 42.856 \\
\hline \multicolumn{11}{|c|}{ Beta-adrenergic blockers } \\
\hline \multirow{4}{*}{21.} & \multirow{4}{*}{ Propranolol } & & 10 & 29 & 10 & 18.48 & 108.9 & 90.42 & 5.892 & 489.285 \\
\hline & & & 20 & 27 & 10 & 16.45 & 72.6 & 56.15 & 4.413 & 341.337 \\
\hline & & 4 & 40 & 5 & 10 & 31 & 49 & 18 & 1.580 & 58.064 \\
\hline & & & 80 & 2 & 10 & 55 & 58.33 & 3.33 & 1.06 & 6.054 \\
\hline 22. & Metoprolol & 1 & 25 & 48 & 10 & 15 & 116 & 101 & 7.733 & 673.333 \\
\hline 23. & Atenolol & 1 & 50 & 35 & 10 & 5.12 & 25.97 & 20.85 & 5.072 & 407.226 \\
\hline Alp & +beta adrenerg & & & & & & & & & \\
\hline & I ahetalol & 2 & 50 & 4 & 10 & 50 & 134 & 84 & 2.68 & 168 \\
\hline & Labetalol & 2 & 100 & 17 & 10 & 110 & 177.9 & 67.9 & 1.617 & 61.727 \\
\hline & Carvedilol & 1 & 6.25 & 50 & 10 & 12 & 76.85 & 64.85 & 6.404 & 540.416 \\
\hline Alp & -blockers & & & & & & & & & \\
\hline & & & 2.5 & 3 & 10 & 57.5 & 96 & 38.5 & 1.669 & 66.956 \\
\hline & Prazosin & 2 & 5 & 4 & 10 & 85 & 129 & 44 & 1.517 & 51.764 \\
\hline & & & 1 & 2 & 10 & 100 & 182.18 & 82.18 & 1.821 & 82.18 \\
\hline 27. & Terazosin & 3 & 2 & 2 & 10 & 180 & 269.95 & 89.95 & 1.499 & 49.972 \\
\hline & & & 5 & 2 & 10 & 170 & 540 & 370 & 3.176 & 217.647 \\
\hline & & & 1 & 3 & 10 & 20 & 40 & 20 & 2 & 100 \\
\hline 28. & Doxazosin & 3 & 2 & 3 & 10 & 33 & 73.83 & 40.83 & 2.237 & 123.727 \\
\hline & & & 4 & 2 & 10 & 87 & 99.5 & 12.5 & 1.143 & 14.367 \\
\hline & Central sympa & & & & & & & & & \\
\hline 29. & Clonidine & 1 & 100 & 2 & 10 & 13.75 & 20.03 & 6.28 & 1.456 & 45.672 \\
\hline & Metbyldong & 2 & 250 & 6 & 10 & 24.15 & 71.4 & 47.25 & 2.956 & 195.652 \\
\hline & Methyldopa & 2 & 500 & 2 & 10 & 46.8 & 99 & 52.2 & 2.115 & 111.538 \\
\hline & Calcium chan & & & & & & & & & \\
\hline & & & 40 & 6 & 10 & 5 & 8.86 & 3.86 & 1.772 & 77.2 \\
\hline 31. & Verapamil & 3 & 120 & 2 & 10 & 38.5 & 42.34 & 3.84 & 1.099 & 9.974 \\
\hline & & & 240 & 2 & 10 & 75.8 & 83.38 & 7.58 & 1.1 & 10 \\
\hline & & & 30 & 11 & 10 & 19.47 & 26.7 & 7.23 & 1.371 & 37.134 \\
\hline 32. & Diltiazem & 3 & 60 & 9 & 10 & 38.38 & 54.54 & 16.16 & 1.421 & 42.105 \\
\hline & & & 90 & 5 & 10 & 66.57 & 104.16 & 37.59 & 1.564 & 56.466 \\
\hline 33. & Nifedipine & 1 & 5 & 3 & 10 & 12.88 & 14.55 & 1.67 & 1.129 & 12.965 \\
\hline
\end{tabular}

Continued. 


\begin{tabular}{|c|c|c|c|c|c|c|c|c|c|c|}
\hline $\begin{array}{l}\dot{\theta} \\
\dot{z}\end{array}$ & 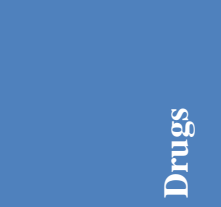 & 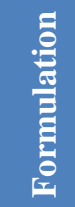 & 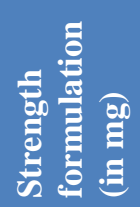 & 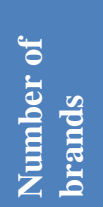 & 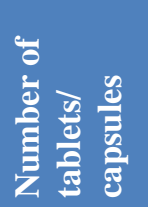 & 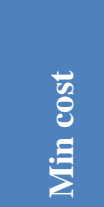 & 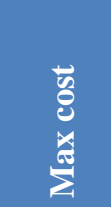 & ن & 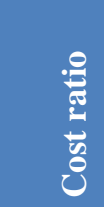 & 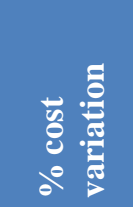 \\
\hline 34. & Felodipine & 1 & 5 & 2 & 10 & 62 & 115.56 & 53.56 & 1.863 & 86.387 \\
\hline 35. & Amlodipine & 1 & 5 & 93 & 10 & 0.025 & 97.1 & $\begin{array}{l}97.07 \\
5\end{array}$ & 3884 & 388300 \\
\hline \multirow{2}{*}{36.} & \multirow{2}{*}{ Cilnidipine } & \multirow{2}{*}{2} & 10 & 64 & 10 & 25 & 125.1 & 100.1 & 5.004 & 400.4 \\
\hline & & & 20 & 42 & 10 & 39.3 & 157.7 & 118.4 & 4.012 & 301.272 \\
\hline 37. & Lacidipine & 1 & 4 & 2 & 10 & 68.5 & 75.1 & 6.6 & 1.096 & 9.63 \\
\hline 38. & Lercanidipine & 1 & 10 & 5 & 10 & 25.65 & 93 & 67.35 & 3.625 & 262.573 \\
\hline & \multirow{2}{*}{ Benidipine } & \multirow{2}{*}{2} & 4 & 24 & 10 & 34.65 & 106 & 71.35 & 3.059 & 205.916 \\
\hline & & & 8 & 23 & 10 & 59 & 179 & 120 & 3.033 & 203.389 \\
\hline & Vasodilators & & & & & & & & & \\
\hline 40. & Hydralazine & 1 & 25 & 3 & 10 & 62.5 & 95.23 & 32.73 & 1.523 & 52.368 \\
\hline 41. & Minoxidil & 1 & 5 & 5 & 10 & 150 & 366 & 216 & 2.44 & 144 \\
\hline
\end{tabular}

Table 2: Cost variation analysis of two drug FDCs of antihypertensive drugs.

\begin{tabular}{|c|c|c|c|c|c|c|c|c|c|c|}
\hline $\begin{array}{l}\dot{2} \\
\dot{s}\end{array}$ & 品 & 章 & 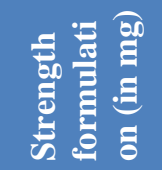 & 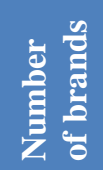 & 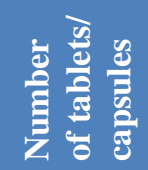 & 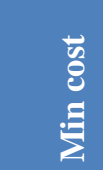 & 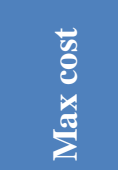 & نे & 递 & 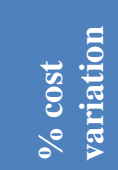 \\
\hline \multirow[b]{2}{*}{1.} & \multirow[b]{2}{*}{ Amlodipine+atenolol } & \multirow[b]{2}{*}{2} & $5+25$ & 10 & 10 & 25.58 & 55 & 29.42 & 2.150 & 115.011 \\
\hline & & & $5+50$ & 98 & 10 & 6.25 & 175.2 & 168.95 & $\begin{array}{l}28.03 \\
2\end{array}$ & 2703.2 \\
\hline \multirow{2}{*}{2.} & \multirow{2}{*}{ Amlodipine+losartan } & \multirow{2}{*}{2} & $2.5+25$ & 2 & 10 & 59.35 & 92.5 & 33.15 & 1.558 & 55.855 \\
\hline & & & $5+50$ & 33 & 10 & 15 & 155.7 & 140.7 & 10.38 & 938 \\
\hline \multirow{2}{*}{3.} & \multirow{2}{*}{ Amlodipine+metoprolol } & \multirow{2}{*}{2} & $2.5+25$ & 15 & 10 & 59 & 135.2 & 76.2 & 2.291 & 129.152 \\
\hline & & & $5+25$ & 37 & 10 & 21 & 149.2 & 128.2 & 7.104 & 610.476 \\
\hline \multirow{2}{*}{4.} & \multirow{2}{*}{ Amlodipine+olmesartan } & \multirow{2}{*}{2} & $5+20$ & 52 & 10 & 45 & 176 & 131 & 3.911 & 291.111 \\
\hline & & & $5+40$ & 26 & 10 & 70 & 239.8 & 169.8 & 3.425 & 242.571 \\
\hline \multirow{2}{*}{5.} & \multirow{2}{*}{$\begin{array}{l}\text { Amlodipine+hydrochlor } \\
\text { othiazide }\end{array}$} & \multirow{2}{*}{2} & $2.5+12.5$ & 7 & 10 & 51 & 76.5 & 25.5 & 1.5 & 50 \\
\hline & & & $5+12.5$ & 14 & 10 & 16.5 & 101.75 & 85.25 & 6.166 & 516.666 \\
\hline \multirow{5}{*}{6.} & \multirow{5}{*}{ Amlodipine+telmisartan } & \multirow{5}{*}{5} & $5+40$ & 142 & 10 & 14.87 & 169.4 & 154.53 & 11.39 & $\begin{array}{l}1039.20 \\
6\end{array}$ \\
\hline & & & $5+80$ & 36 & 10 & 82.5 & 267.5 & 185 & 3.242 & 224.242 \\
\hline & & & $80+5$ & 2 & 10 & 111 & 130 & 19 & 1.171 & 17.117 \\
\hline & & & $2.5+40$ & 9 & 10 & 78.5 & 109.3 & 30.8 & 1.392 & 39.235 \\
\hline & & & $5+40$ & 9 & 10 & 20 & 144.15 & 124.15 & 7.207 & 620.75 \\
\hline 7. & Amlodipine+enalapril & 1 & $5+5$ & 4 & 10 & 33.71 & 94.66 & 60.95 & 2.808 & 180.806 \\
\hline \multirow{2}{*}{8.} & \multirow{2}{*}{ Amlodipine+lisinopril } & \multirow{2}{*}{2} & $2.5+2.5$ & 2 & 10 & 45.5 & 85.15 & 39.65 & 1.871 & 87.142 \\
\hline & & & $5+5$ & 16 & 10 & 49.81 & 130 & 80.19 & 2.609 & 160.991 \\
\hline 9. & $\begin{array}{l}\text { Amlodipine+indapamid } \\
\text { e }\end{array}$ & 1 & $5+1.5$ & 2 & 10 & 113.9 & 122 & 8.1 & 1.071 & 7.111 \\
\hline 10. & Amlodipine+perindopril & 1 & $5+4$ & 2 & 10 & 157 & 170 & 13 & 1.082 & 8.280 \\
\hline \multirow{3}{*}{11.} & \multirow{3}{*}{$\begin{array}{l}\text { Hydrochlorthiazide+bis } \\
\text { oprolol }\end{array}$} & \multirow{3}{*}{3} & $6.25+2.5$ & 5 & 10 & 42.53 & 80.66 & 38.13 & 1.896 & 89.654 \\
\hline & & & $12.5+5$ & 4 & 10 & 46.25 & 135.09 & 88.84 & 2.920 & 192.086 \\
\hline & & & $6.25+5$ & 4 & 10 & 75 & 117.81 & 42.81 & 1.570 & 57.08 \\
\hline \multirow{2}{*}{12.} & \multirow{2}{*}{$\begin{array}{l}\text { Hydrochlorothiazide+ol } \\
\text { mesartan }\end{array}$} & \multirow[t]{2}{*}{2} & $12.5+20$ & 56 & 10 & 45 & 174.19 & 129.19 & 3.870 & $\begin{array}{l}287.088 \\
8\end{array}$ \\
\hline & & & $12.5+40$ & 46 & 10 & 75 & 323.7 & 248.7 & 4.316 & 331.6 \\
\hline \multirow{2}{*}{13.} & \multirow{2}{*}{$\begin{array}{l}\text { Hydrochlorthiazide+losa } \\
\text { rtan }\end{array}$} & \multirow{2}{*}{2} & $12.5+25$ & 2 & 10 & 47.3 & 88 & 40.7 & 1.860 & 86.046 \\
\hline & & & $12.5+50$ & 69 & 10 & 30 & 165.6 & 135.6 & 5.52 & 452 \\
\hline
\end{tabular}




\begin{tabular}{|c|c|c|c|c|c|c|c|c|c|c|}
\hline $\begin{array}{l}\dot{\theta} \\
\dot{\hbar}\end{array}$ & 题 & 章 & 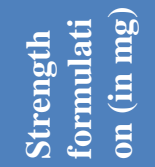 & 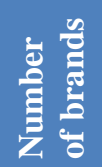 & 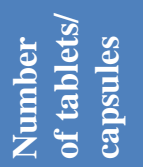 & $\begin{array}{l}\vec{y} \\
8 \\
\vdots \\
\vdots\end{array}$ & 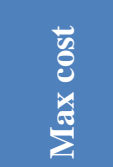 & 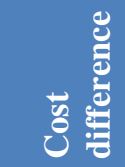 & 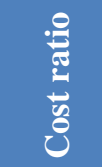 & 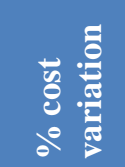 \\
\hline 14. & Ramipril+losartan & 1 & $5+50$ & 3 & 10 & 80.76 & 152.25 & 71.49 & 1.885 & 88.521 \\
\hline 15. & $\begin{array}{l}\text { Hydrochlorthiazide+ena } \\
\text { lapril }\end{array}$ & 1 & $25+10$ & 7 & 10 & 32 & 90.48 & 58.48 & 2.827 & 182.75 \\
\hline 16. & $\begin{array}{l}\text { Hydrochlorthiazide+irbe } \\
\text { sartan }\end{array}$ & 1 & $12.5+150$ & 2 & 10 & 157 & 225.2 & 68.2 & 1.434 & 43.439 \\
\hline 17. & $\begin{array}{l}\text { Spironolactone+furosem } \\
\text { ide }\end{array}$ & & $50+20$ & 6 & 10 & 27 & 67.15 & 40.15 & 2.487 & 148.703 \\
\hline \multirow{4}{*}{18.} & \multirow{4}{*}{$\begin{array}{l}\text { Spironolactone+torsemi } \\
\text { de }\end{array}$} & \multirow{4}{*}{4} & $25+10$ & 4 & 10 & 24 & 34.35 & 10.35 & 1.431 & 43.125 \\
\hline & & & $50+10$ & 11 & 10 & 40 & 103.8 & 63.8 & 2.595 & 159.5 \\
\hline & & & $50+20$ & 6 & 10 & 45 & 134 & 89 & 2.977 & 197.777 \\
\hline & & & $50+5$ & 4 & 10 & 35 & 43.6 & 8.6 & 1.245 & 24.571 \\
\hline 19 & Atenolol+indapamide & 1 & $50+2.5$ & 3 & 10 & 52.8 & 89.8 & 37 & 1.700 & 70.075 \\
\hline
\end{tabular}

Table 3: Cost variation analysis of three drug FDCs of antihypertensive drugs.

\begin{tabular}{|c|c|c|c|c|c|c|c|c|c|c|}
\hline $\begin{array}{l}\dot{\theta} \\
\dot{Z} \\
\dot{0}\end{array}$ & $\stackrel{e_{0}^{\circ}}{2}$ & 言 & 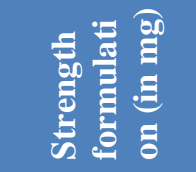 & 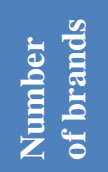 & 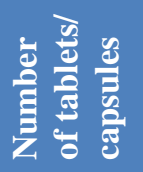 & $\begin{array}{l}\overrightarrow{\tilde{g}} \\
\stackrel{\Xi}{\Sigma}\end{array}$ & 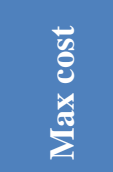 & 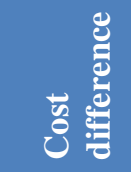 & 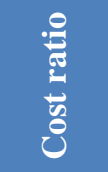 & 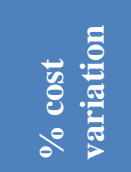 \\
\hline 1. & $\begin{array}{l}\text { Amlodipine+losartan+ } \\
\text { hydrochlorthiazide }\end{array}$ & 1 & $5+50+12.5$ & 6 & 10 & 87.35 & 142.5 & 55.15 & 1.631 & 63.136 \\
\hline \multirow{2}{*}{2.} & \multirow{2}{*}{$\begin{array}{l}\text { Olmesartan+amlodipne } \\
\text { +chlorthalidone }\end{array}$} & \multirow{2}{*}{2} & $20+5+12.5$ & 5 & 10 & 104.15 & 143.55 & 39.4 & 1.378 & 37.830 \\
\hline & & & $40+5+12.5$ & 4 & 10 & 181.3 & 214.5 & 33.2 & 1.183 & 18.312 \\
\hline \multirow{2}{*}{3.} & \multirow{2}{*}{$\begin{array}{l}\text { Olmesartan+amlodipin } \\
\text { e+hydrochlorthiazide }\end{array}$} & \multirow{2}{*}{2} & $20+5+12.5$ & 22 & 10 & 69.3 & 192.32 & 123.02 & 2.775 & 177.518 \\
\hline & & & $40+5+12.5$ & 16 & 10 & 121 & 266.7 & 145.7 & 2.204 & 120.413 \\
\hline 4. & $\begin{array}{l}\text { Telmisartan+benidipin } \\
\text { e+chlorthalidone }\end{array}$ & 1 & $40+4+12.5$ & 3 & 10 & 139 & 179 & 40 & 1.287 & 28.776 \\
\hline \multirow{2}{*}{5.} & \multirow{2}{*}{$\begin{array}{l}\text { Telmisartan+cilnidipin } \\
\text { e+metoprolol }\end{array}$} & \multirow{2}{*}{2} & $40+10+25$ & 4 & 10 & 99 & 150 & 51 & 1.515 & 51.515 \\
\hline & & & $40+10+50$ & 3 & 10 & 110 & 178.5 & 68.5 & 1.622 & 62.272 \\
\hline \multirow{4}{*}{6.} & \multirow{4}{*}{$\begin{array}{l}\text { Telmisartan+amlodipin } \\
\text { e+chlorthalidone }\end{array}$} & \multirow{4}{*}{4} & $40+5+12.5$ & 10 & 10 & 100 & 150 & 50 & 1.5 & 50 \\
\hline & & & $40+5+6.25$ & 4 & 10 & 90 & 114 & 24 & 1.266 & 26.666 \\
\hline & & & $80+5+12.5$ & 7 & 10 & 160 & 197.63 & 37.63 & 1.235 & 23.518 \\
\hline & & & $80+5+6.5$ & 3 & 10 & 164.95 & 178 & 13.05 & 1.079 & 7.911 \\
\hline & \multirow{2}{*}{$\begin{array}{l}\text { Telmisartan+amlodipin } \\
\text { e+hydrochlorthiazide }\end{array}$} & \multirow[t]{2}{*}{2} & $40+5+12.5$ & 52 & 10 & 20 & 181.5 & 161.5 & 9.075 & 807.5 \\
\hline & & & $80+5+12.5$ & 9 & 10 & 151 & 188 & 37 & 1.245 & 24.503 \\
\hline \multirow{3}{*}{8.} & \multirow{3}{*}{$\begin{array}{l}\text { Telmisartan+cilnidipin } \\
\text { e+chlorthalidone }\end{array}$} & \multirow{3}{*}{3} & $40+10+12.5$ & 22 & 10 & 100 & 189 & 89 & 1.89 & 89 \\
\hline & & & $40+10+6.25$ & 20 & 10 & 82.5 & 176 & 93.5 & 2.133 & 113.333 \\
\hline & & & $40+20+12.5$ & 2 & 10 & 140 & 156.32 & 16.32 & 1.116 & 11.657 \\
\hline & $\begin{array}{l}\text { Telmisartan+chlorthali } \\
\text { done+metoprolol }\end{array}$ & 1 & $40+6.25+50$ & 2 & 10 & 120 & 141 & 21 & 1.175 & 17.5 \\
\hline
\end{tabular}

Table 4: Comparison of maximum cost of essential antihypertensives (as per NLEM) with their ceiling price (as per DPCO).

\begin{tabular}{|llllllll|}
\hline $\begin{array}{l}\text { Sr. } \\
\text { No. }\end{array}$ & Medicine & $\begin{array}{l}\text { Dosage } \\
\text { (in } \mathbf{m g})\end{array}$ & $\begin{array}{l}\text { No. of } \\
\text { tablet }\end{array}$ & $\begin{array}{l}\text { Ceiling price per } \\
\text { tablet/capsule }\end{array}$ & $\begin{array}{l}\text { Ceiling price per 10 } \\
\text { tablets/capsules }\end{array}$ & $\begin{array}{l}\text { Maximum } \\
\text { cost }\end{array}$ & $\begin{array}{l}\text { Cost } \\
\text { difference }\end{array}$ \\
\hline 1. & Amlodipine & 5 & 1 & 2.60 & 26 & 97.1 & 71.1 \\
\hline 2. & Atenolol & 50 & 1 & 1.78 & 17.8 & 25.97 & 8.17 \\
\hline \multirow{2}{*}{ 3. } & \multirow{2}{*}{ Enalapril } & 2.5 & 1 & 1.93 & 19.3 & 21.54 & 2.24 \\
\hline 4. & & 5 & & 3.2 & 32 & 32.31 & 0.31 \\
\hline
\end{tabular}




\begin{tabular}{|llllllll|}
\hline $\begin{array}{l}\text { Sr. } \\
\text { No. }\end{array}$ & Medicine & $\begin{array}{l}\text { Dosage } \\
\text { (in mg) }\end{array}$ & $\begin{array}{l}\text { No. of } \\
\text { tablet }\end{array}$ & $\begin{array}{l}\text { Ceiling price per } \\
\text { tablet/capsule }\end{array}$ & $\begin{array}{l}\text { Ceiling price per 10 } \\
\text { tablets/capsules }\end{array}$ & $\begin{array}{l}\text { Maximum } \\
\text { cost }\end{array}$ & $\begin{array}{l}\text { Cost } \\
\text { difference }\end{array}$ \\
\hline & $\begin{array}{l}\text { Hydrochlorot } \\
\text { hiazide }\end{array}$ & 25 & 1 & 1.68 & 16.8 & 10.97 & 5.63 \\
\cline { 3 - 9 } & 50 & 1 & 0.08 & 0.8 & 10.97 & 10.17 \\
\hline 5. & \multirow{2}{*}{ Methyldopa } & 250 & 1 & 2.44 & 24.4 & 71.4 & 47 \\
\cline { 3 - 9 } & 500 & 1 & 4.67 & 46.7 & 99 & 52.3 \\
\hline 6. & Ramipril & 2.5 & 1 & 4.72 & 47.2 & 52.86 & 5.66 \\
\hline \multirow{2}{*}{ 7. } & \multirow{2}{*}{ Telmisartan } & 20 & 1 & 3.64 & 36.4 & 61.5 & 25.1 \\
\cline { 3 - 9 } & & 40 & 1 & 6.57 & 65.7 & 59.6 & -6.1 \\
\hline
\end{tabular}

\section{DISCUSSION}

In this study, noticeable cost variation was found in different brands of same antihypertensive drugs. The cost variation observed in the present study was as high as $388300 \%$ of amlodipine, $2703 \%$ (amlodipine+atenolol), 990.90\% (furosemide), $769.64 \%$ (losartan). Other significantly high cost variations found in the present study were $\quad 1039.20 \%$ (amlodipine+telmisartan), $938 \%$ (amlodipine+losartan), $\quad 807.5 \% \quad$ (telmisartan +amlodipine+hydrochlorthiazide) (Table 2 and 3).

Similar results were found in other studies. The study done by Ahmed et al stated high as $2337.50 \%$ for hydralazine. $1315.25 \%$ (telmisartan+hydrochlorthiazide), $870.58 \%$ (amlopdipine), 558.34\% (amlodipine+atenolol), 537.68\% (valsartan), 394.44\% (metoprolol), 344.44\% (enalapril), $316.22 \%$ (propranolol), $300 \%$ (lisinopril). ${ }^{14}$

The other study done by Karve et al reveal that the prices of most of the antihypertensive brands have percentage price variation above $100 \% .^{15}$

The reasons for this price variation could be as follows: the existing market structure of the pharmaceutical industry, asymmetry of information or imperfect information, government regulations and pricing policies, costs of raw supplies, distribution and promotion and economic goals of the parent company, target return on investment. ${ }^{15}$

At present, there are very few medicines under drug prices control order. Hence, it is necessary that the government of India should bring all lifesaving and essential medicines under price control. FDCs of antihypertensive drugs are not included in NLEM which should be taken into consideration while revising the list. Due consideration must be placed on the pricing of drugs in the NLEM to increase their accessibility to common people. DPCO appears to be an effective tool to keep in rein the drug prices which should be implemented for all drugs included in NLEM.

Despite increased awareness, poor adherence to treatments for chronic diseases remains a global problem. Adherence issues are common in patients taking antihypertensive drug therapy and are associated with increased risks of coronary and cerebrovascular events. To gain the maximal benefits of their antihypertensive therapy, it is important for the clinicians to support adherence to prescribed drugs. Because hypertension is so common and its treatment often requires the use of more than one medication, antihypertensive drug therapy is a common target of costcutting efforts.

While patients may assume that their doctors know the cost of the drugs they are prescribing, that's often not the case. Doctors in clinics seeing patients don't necessarily know how much the drugs cost. Doctors must prescribe rationally. Rational prescribing implies using the right drug for the right patient at the right time in the right dose and manner of administration, at affordable cost and with right information. They need to be educated about the cost variation in different brands of same drug.

\section{CONCLUSION}

This study highlights that there is a huge price variation among the antihypertensive drugs manufactured by different companies. Some measures must be taken by the government to bring about the uniformity in the price. It will help to reduce the economic burden on the patients to some extent. There is a strong need to create awareness about this huge price variation among the general public, health care providers, healthcare payers, government agencies, policy makers, pharmacists for appropriate intervention to reduce economic burden on patients as well as on the healthcare system.

\section{Funding: No funding sources}

Conflict of interest: None declared

Ethical approval: The study was approved by the Institutional Ethics Committee

\section{REFERENCES}

1. GBD 2016 Risk Factors Collaborators. Global, regional, and national comparative risk assessment of 84 behavioral, environmental and occupational, and metabolic risks or clusters of risks, 1990-2016: a systematic analysis for the global burden of disease study 2016. Lancet. 2017;390(10100):1345-422.

2. Kearney PM, Whelton M, Reynolds K, Muntner P, Whelton PK, He J. Global burden of hypertension: 
Analysis of worldwide data. Lancet. 2005;365(9455):217-23.

3. Gupta R, Xavier D. Hypertension: the most important non communicable disease risk factor in India. Indian Heart J. 2018;70(4):565-72.

4. Zhou D, Bo X, Zhao M, Wang L, Veeranki S. Uncontrolled hypertension increases risk of all-cause and cardiovascular disease mortality in US adults: the NHANES III linked mortality study. Sci Reps. 2018;8:9418.

5. Gupta R, Yusuf S. Towards better hypertension management in India. Indian $\mathrm{J}$ Med Res. 2014;139(5):657-60.

6. Kardas P, Bishai WR. Compliance in infective medicine. Adv Stud Med. 2006;6(7C):652-8.

7. Das SC, Mandal M, Mandal SC. A critical study on availability and price variation between different brands: impact on access to medicines. Indian J Pharm Sci. 2007;69(1):160-3.

8. Piette JD, Heisler M, Wagner TH. Cost-related medication underuse among chronically ill adults: The treatments people forgo, how often, and who is at risk. Am J Public Health. 2004;94:1782-7.

9. Kumar V, Gupta NV, Kumar KA. A comparison between old and latest systems in DPCO. Int J Pharm Pharmaceut Sci. 2014;6(2):19-20.

10. Government of India Ministry of Chemicals and Fertilizers Department of Pharmaceuticals National Pharmaceutical Pricing Authority. Available at: https://www.nppaindia.nic.in/wpcontent/uploads/2020/03/1-1.pdf.

11. Rivers PA, Glover SH. Health care competition, strategic mission, and patient satisfaction: research model and propositions. J Health Organ Manag. 2008;22(6):627-641.

12. Mir SA. A calm look at the cost of various brands of antiasthmatic drugs available in India. Int J Basic Clin Pharmacol. 2016;5:142-5.

13. Central Drugs Standard Control Organization. Fact sheet: National List of Essential Medicines, 2015. Available at: https://cdsco.gov.in/opencms/opencms/en/consumer/ Essential-Medicines/. Accessed on 15 May 2021.

14. Mir SA. Cost variation analysis of different brands of commonly prescribed antihypertensive drugs, available in Indian market: a pharmacoeconomic study. Int J Basic Clin Pharmacol. 2018;7(3):556-60.

15. Karve AV, Chattar KB. Cost analysis study of oral antihypertensive agents available in Indian market. Int J Basic Clin Pharmacol. 2014;3(3):479-83.

Cite this article as: Upasani PC, Barve RM, Hiray RS. A pharmacoeconomic study of different brands of commonly prescribed antihypertensive drugs. Int $\mathbf{J}$ Basic Clin Pharmacol 2021;10:976-83. 